\title{
Perspectives on the prevention and treatment of infection for orthopedic tissue engineering applications
}

\author{
SHAH Sarita $\mathrm{R}^{1}$, KASPER F Kurtis ${ }^{1} \&$ MIKOS Antonios $\mathrm{G}^{1,2^{*}}$ \\ ${ }^{1}$ Department of Bioengineering, Rice University, P.O. Box 1892, MS 142, Houston, Texas, 77251-1892, USA; \\ ${ }^{2}$ Department of Chemical and Biomolecular Engineering, Rice University, P.O. Box 1892, MS 142, Houston, Texas, 77251-1892, USA
}

Received November 15, 2012; accepted February 20, 2013; published online June 20, 2013

\begin{abstract}
The emergence of orthopedic tissue engineering devices as viable clinical solutions demands that the field address significant clinical complications associated with the implantation of foreign materials in the body, especially infection. While tissue engineering has focused on the development of methods to regenerate and repair, until recently there has been a relative dearth of literature regarding the intersection of tissue engineering and infection. In particular, local delivery of antimicrobials has long been of clinical interest, but only recently has that been translated into the realm of tissue engineering. In this perspective, we briefly review major modes of local delivery for infection prevention and treatment and discuss possible strategies for preventing implant-associated infections.
\end{abstract}

tissue engineering, infection, antibiotic delivery, antifouling agents

Citation: Shah S R, Kasper F K, Mikos A G. Perspectives on the prevention and treatment of infection for orthopedic tissue engineering applications. Chin Sci Bull, 2013, 58: 4342-4348, doi: 10.1007/s11434-013-5780-8

In recent years, the field of tissue engineering has made great strides in the development of implantable materials to aid in the repair and regeneration of defects due to pathology or injury. However, infectious complications remain a significant source of morbidity, and even mortality, in patients receiving implants of any kind, and infection rates have remained stable over the past decades. The infection rate in total hip and knee replacements, a procedure that surgeons have extensive experience with and that is performed in a sterile surgical field, is still $1 \%-2 \%$, and the consequent treatment and removal can cause considerable morbidity $[1,2]$. Patients experience pain, loss of productivity, and decrease in quality of life, and the cost of device-related infection is estimated to be over $\$ 1$ billion annually [3]. As orthopedic tissue engineering implants become more clinically implemented, it is reasonable to expect that they will face similar challenges with respect to infectious complications.

The interaction between bacteria and implanted bio-

*Corresponding author (email: mikos@rice.edu) materials has long been known and researched, but methods to deal with implant-associated infections remain limited. In 1987, Gristina [4] coined the phrase "race for the surface" to describe the competition between cells and bacteria for colonization of a surface. Bacteria are inherently favored in this race, given their natural ability to colonize both biological and non-biological surfaces. Implants that fail to integrate with the host tissue are particularly vulnerable to hematogenous seeding from distant infection sites over the entire lifetime of the implant. In addition, when freefloating planktonic bacteria adhere to a surface, they can form biofilms, a hardy microenvironment consisting of proteins, extracellular DNA, and polysaccharides that allow bacteria to adhere to surfaces, modulate host immune response, and enter a quiescent state of lower metabolic activity while providing protection against antimicrobial agents [5]. Biofilms pose a considerable threat to implants in both the acute and chronic setting, as delayed infections can be seen several years after implantation [6]. Ideally, implanted materials would be toxic specifically to bacterial cells, prevent the adhesion of bacteria, block the phenotypic changes 
between planktonic and surface-adhered bacteria that result in biofilm formation, and promote host tissue integration to prevent future colonization with bacteria. While the problems presented by implant-associated infection are well known, there is still a marked lack of progress in the area of suitable antimicrobial materials and coatings. Compounding the problem, the United States faces rising rates of antibiotic-resistant bacterial strains in conjunction with decreasing rates of new antimicrobial drug discovery and marketing [7].

Tissue engineers face several challenges in regards to implant-associated infection: (1) develop anti-microbial or drug-delivering materials to treat infection while minimizing antibacterial resistance, (2) combat the growth of biofilms on the implant, and (3) adapt to the emergence of antibiotic-resistant strains.

\section{Clinical significance and management}

Infection is known to inhibit healing in several tissue types, including bone, gastrointestinal epithelium, and cutaneous epithelium [8-10]. Physiologic wound healing in response to injury occurs in four sequential and timed phases: hemostasis, inflammation, proliferation and repair, and remodeling. Rapid hemostasis occurs via formation of a fibrin clot to control bleeding. The injured tissue then recruits inflammatory cells through release of pro-inflammatory cytokines and growth factors, resulting in the sequential infiltration of neutrophils, macrophages, and lymphoctyes. Neutrophils play a critical role in controlling infection during this stage through production of reactive oxidative species, while macrophages clear pro-inflammatory debris. After damaged and necrotic tissue has been cleared, the defect is repaired through cell proliferation and synthesis of extracellular matrix. Finally, the repaired tissue enters the remodeling phase, in which the new tissue is modified to approximate normal architecture and strength as closely as possible [11]. Microbes derail normal wound healing by inducing the affected area to remain in the inflammatory phase for a prolonged period of time. If neutrophils are deficient or inadequate to clear bacteria, the presence of bacterial toxins such as lipopolysaccharide (LPS) can induce prolonged and elevated levels of pro-inflammatory cytokines such as IL-1 and TNF- $\alpha$ [11]. Recent studies have shown that the principal causative organism for osteomyelitis, Staphylococcus aure$u s$, is capable of upregulating production of several pro-inflammatory cytokines and inducing resorption of bone through RANK-L-mediated osteoclastogenesis, resulting in delayed or non-union of the fracture $[8,12]$. Similarly, formation of bacterial biofilms in a cutaneous wound has been shown to result in non-healing in a murine model [10]. Interestingly, the wounds were able to re-epithelialize if the biofilms were disrupted [10]. Though the mechanisms by which infection inhibits healing are just beginning to be elucidated, it is obvious that tissue engineering strategies will fail without adequate prevention and treatment of infection.

Currently, orthopedic infections are treated with systemic antibiotics, delivered either orally or parenterally. Systemic antibiotics can be supplemented with antibiotics delivered locally through surgically implanted poly(methyl methacrylate) (PMMA) beads. Surgical irrigation and debridement can also be attempted. Pre-clinical animal studies show that early irrigation and debridement, especially in concert with antibiotics, may prevent infection, but clinical studies show that the failure rate of this approach is high if the site is already infected [13-16]. If the infection is persistent, removal or replacement of the implant becomes necessary. Implant failure due to infection leaves both the patient and physician in a difficult situation: either the implant will not be replaced or a new implant must be placed in a contaminated area. Solutions to this problem must be two-fold: development of materials that deter initial infection from taking place and materials that are capable of treating already existing infection. Local delivery of antimicrobial drugs and development of antimicrobial and antibiofilm materials appears to be a promising approach that has undergone significant evolution in the past twenty to thirty years. In this perspective, we will discuss the major modes of delivery that have emerged as possible solutions to the problem of infection at implant sites.

\section{Current research in infection}

\subsection{Local antibiotic delivery}

Local delivery of antibiotics is an attractive option for the prevention of infection for many reasons. First, a large dose of antibiotics can be delivered directly to the site of infection, circumventing the need for areas with good blood flow. This is of particular benefit in patients with diabetes, vasculopathy, or severe trauma. Second, antibiotic concentration in the infected area is more likely to be in the therapeutic range, reducing the chance of breeding antibiotic resistant strains. Third, systemic side effects are reduced or eliminated since antibiotics should not reach high serum concentrations. Considerations that are important when evaluating the utility and desirability of a delivery system include delivery kinetics and toxicity of the agent locally. Generally, sustained release in the therapeutic range for an antibiotic/bacterial species combination is desired in order to guard against infection until native vascularity can be restored. Although high concentrations are useful for treatment, antibiotics can also be toxic to mammalian cells. Rathbone et al. [17] demonstrated in vitro that several antibiotics commonly used for local delivery in osteomyelitis are toxic to osteoblasts. Though there are clear benefits of local delivery, there is not yet a consensus on the optimal strategy or material that should be employed. 
Both natural and synthetic materials have been investigated as potential local delivery vehicles. Natural polymers such as collagen and chitosan are simply and rapidly loaded by soaking them in a solution of the desired antibiotic. Collagen and chitosan both deliver antibiotics in a rapid "burst" and release is normally complete within $4 \mathrm{~d}[18,19]$. These short-duration release kinetics may not be appropriate for surgically implanted delivery vehicles, but they may be useful for cutaneous applications, such as infection control for burn wounds [20].

Antibiotic-impregnated PMMA beads have been used clinically to deliver antibiotics locally for at least 30 years [21]. They are fabricated at the time of implantation by mixing the desired antibiotic with PMMA and forming the resulting mixture into beads. The "standard" formulation is $1 \mathrm{~g}$ antibiotic mixed into a $40 \mathrm{~g}$ package of bone cement, although this ratio can be altered. These beads have the advantage of clinical familiarity, and in vivo studies have indicated that they are effective at eradicating acute infections in long bones [22]. In addition, the antibiotic is easily varied based on the most likely pathogen. However, since PMMA is hydrophobic and non-biodegradable, water does not swell the material to release antibiotics, and antibiotics incorporated into the interior of the bead may not be released. Antibiotic release from PMMA may be as low as $25 \%$ to $50 \%$ of total incorporated antibiotic. Release kinetics are widely variable between drug classes, and only clindamycin and vancomycin can be eluted at therapeutic levels at $28 \mathrm{~d}$ [21]. Since PMMA is non-biodegradable, a second surgery is required to remove the beads once antibiotic elution has stopped. Recent studies have also shown that bacterial biofilms can persist on antibiotic-eluting cement beads when implanted into infected areas [23]. In light of these issues, biodegradable materials have been investigated as possible antibiotic carriers.

Calcium sulfate is a resorbable ceramic material that has been studied as an antibiotic carrier. Similar to PMMA, these beads are fabricated by incorporating the antibiotic into a calcium sulfate kit, so the antibiotic can be varied easily. Tobramycin release has been shown to occur at least up to $14 \mathrm{~d}$, but levels are sub-therapeutic by $28 \mathrm{~d}$ [24]. Resorption time from different studies varied from 1 to 6 months and can be somewhat tuned by varying the density of calcium sulfate crystals [21]. Studies on the efficacy of calcium sulfate as a drug-delivery vehicle are widely varied. A 2010 study shows that calcium sulfate pellets are highly efficacious, with resolution of osteomyelitis in all patients followed [25]. However, a 2009 retrospective study on the use of antibiotic-loaded calcium sulfate in combat-related open fractures was less positive, with a $22 \%$ rate of osteomyelitis, a $25 \%$ rate of heterotopic ossification in patients whose limbs were not eventually amputated, and over half of patients experiencing sterile draining sinuses [26]. However, in comparison to PMMA, calcium sulfate is a more appropriate vehicle for tissue engineering applications since it is resorbable and osteoconductive. Unfortunately, calcium sulfate lacks sufficient mechanical strength to act as a bone scaffold, and the resorption rate of calcium sulfate may be too fast for bone regeneration.

Biodegradable polymers such as poly(lactic acid) (PLA) and poly(lactic-co-glycolic acid) (PLGA) have also been used to locally deliver antibiotics. Unlike PMMA and calcium sulphate, these polymers can be used in several different forms, including coatings, microspheres [27], and electrospun fibers [28]. Release of antibiotics from PLGA microspheres, in particular, has been well-characterized, and encompasses many different classes of antibiotics such as vancomycin [29], nafcillin [30], colistin [27], tobramycin [31], and ciprofloxacin [32]. Antibiotic-loaded PLGA microspheres are a promising vehicle for local delivery given the biodegradable nature and biocompatibility of PLGA as well as its desirable release kinetics. The release kinetics of antibiotics from microspheres are characterized by a burst release followed by slow release and a possible smaller, late burst. However, the release kinetics are altered to a triphasic release if the microspheres are incorporated into a PMMA construct [27].

Biodegradable microspheres present an interesting solution to the problem of antibiotic delivery for tissue engineering applications. Of the vehicles discussed thus far, degradable microspheres are unique in that they can easily be incorporated into constructs of other materials that are capable of bridging to the regeneration of native tissue. Bioactive factors such as vascular endothelial growth factor and bone morphogenetic protein-2 have been successfully incorporated into and released from biodegradable microspheres as well $[33,34]$. Composite scaffolds could serve multiple functions, such as combining antibiotic and growth factor delivery through biodegradable microspheres, and mechanical stability and promotion of tissue growth through the bulk material.

\subsection{Alternatives to conventional antibiotics}

Although this review has thus far focused on the use of biomaterials as antibiotic carriers for tissue engineering applications, discussion of local antimicrobials would be incomplete without mention of alternative antimicrobial methods. As mentioned earlier, the development of microbial resistance to conventional antibiotic therapy continues to be a major clinical challenge [7]. To address this serious issue, research into alternative antimicrobials has gained traction in both historically important areas such as silver compounds and newer areas, such as nanoparticle formulations and novel synthetic antimicrobial peptides.

Silver has been an important antimicrobial since ancient times, when it was most likely used for chronic wounds and ulcers [35]. It has remained a relevant antimicrobial, now being used for burn treatment, catheter coatings, and dental work [35-37]. Silver causes microbial death by penetrating 
the cell wall or membrane and interfering with cell replication and respiration [35,38]. There are several topical formulations of silver used clinically, such as silver nitrate and silver sulfadiazine, but silver nanoparticles (NPs) are gaining popularity, even amongst other types of metal and metal oxide NPs, as an antimicrobial with far-reaching potential since they have been shown to be effective again bacteria, viruses, and eukaryotes [39]. Silver NPs have been used as a local antimicrobial in coatings of medical devices, wound dressings, and textile fabrics [40]. Though silver NPs appear to be an attractive solution to the problem of antibiotic resistance, there are reports of silver-resistant bacterial strains [37]. More importantly, plasmids that encode for silver resistance also have been shown to often encode antibiotic-resistance as well [41]. Increased use of silver NPs may lead to an increased selective pressure toward antibioticresistance bacterial strains through this genetic linkage.

Antimicrobial peptides (AMPs) are short peptides about 20-50 residues in length that are found in many species of plants and animals, including humans [42]. These peptides are generally amphiphilic, allowing them to permeabilize bacterial membranes through a combination of electrostatic and hydrophobic interactions [42]. AMPs are also generally able to distinguish between mammalian and bacterial cell membranes due to the predominance of negatively charged lipids on the surface of bacterial membranes not seen with mammalian cells [42]. An example of a naturally occurring antimicrobial peptide is human lactoferrin 1-11 [45]. Synthetic analogues of these naturally-occurring peptides, such as arylamide foldamers, have been synthesized and shown to be effective against bacteria $[42,43]$. There has been interest in local delivery of AMPs as an alternative to conventional antibiotics. Several studies have described local delivery of AMPs in vitro and in vivo via calcium phosphate carrier [44-46]. These studies show that it is possible to incorporate and locally release AMPs while retaining anti- microbial activity, although broad-spectrum AMPs such as human lactoferrin 1-11 may not be as potent as conventional antibiotics such as gentamicin [44-46]. Shukla et al. [47] incorporated a different AMP, ponericin G1, into a degradable polyectrolyte multilayer film that has applications as coatings for medical devices and wound healing. Controlled release over $10 \mathrm{~d}$ was achieved with tunable release profiles and retention of antimicrobial activity [47]. AMPs have recently become a popular alternative because it was previously believed that resistance to AMPs would be unlikely given that significant changes to the bacterial cell membrane would be required and these peptides have been present in bacterial environments for $10^{8}$ years without evolution of significant resistance [48]. However, some evolutionary biologists have argued that bacteria would be able to evolve resistance mechanisms against these peptides when they are used therapeutically due to the creation of a circumstance in which there is selective pressure for resistant strains [49]. Recent research has shown that bacteria are able to develop resistance in laboratory conditions and several mechanisms by which microbes can become resistant to AMPs have been elucidated $[50,51]$.

\subsection{Antifouling agents}

As the importance of biofilm becomes increasingly recognized, more emphasis is being placed on the development of antifouling polymers and biofilm dispersal agents. Biofilms are capable of growing on the surface of implanted materials and have even been shown to survive on the surface of antibiotic-releasing biomaterials [52,53]. As shown in Figure 1, Staphylococcus aureus is able to form biofilm even on antibiotic-releasing poly(methyl methacrylate) constructs.

Biofilm dispersal agents can either inhibit the formation of biofilm or promote the detachment of biofilm bacteria. The dispersal of implant biofilms has several benefits for
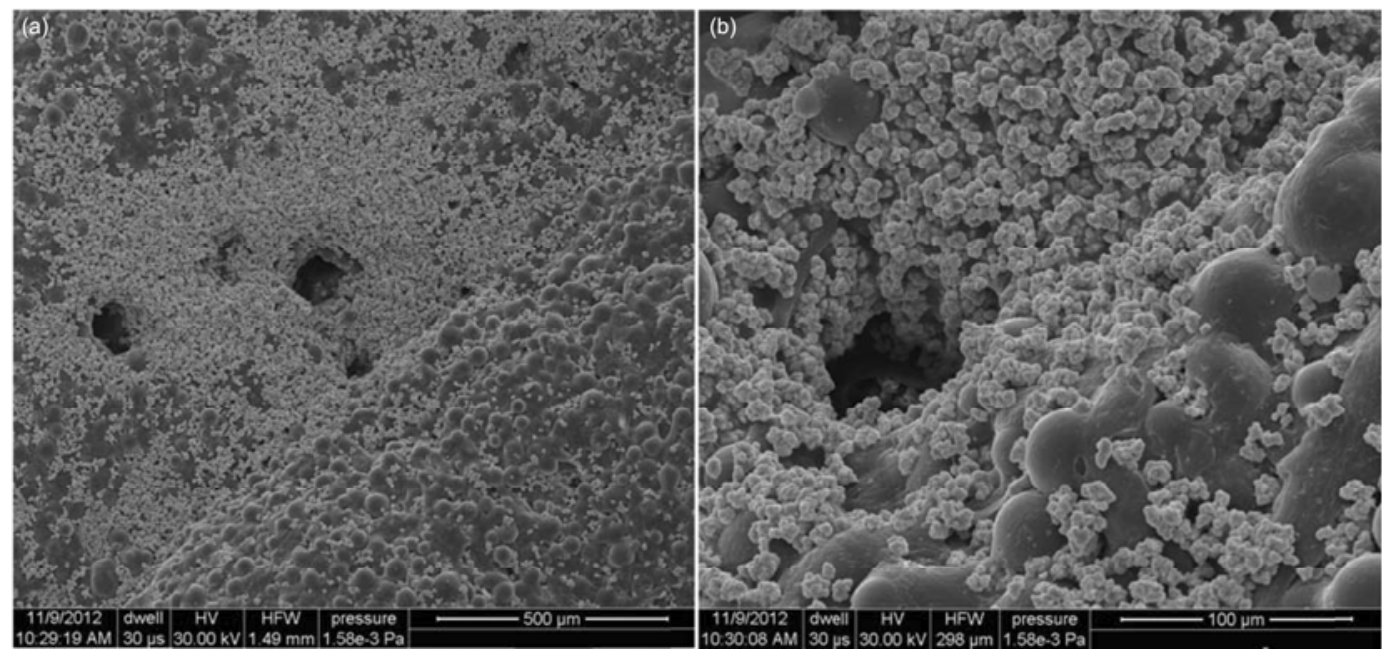

Figure 1 Staphylococcus aureus biofilm formation on vancomycin-loaded porous poly(methyl methacrylate) constructs at $100 \times($ a) and $400 \times(b)$. 
the patient. The dissolution of the biofilm prevents the implant from being a source of delayed infection, as bacteria can detach from the biofilm and seed distant sites. The surface of the implant also becomes more hospitable to native cells through lack of competition with bacteria, a distinct benefit when tissue integration is the goal. Additionally, since biofilm-associated bacteria are known to be much more resistant to antibiotics than planktonic bacteria, forcing biofilm bacteria back into their metabolically active planktonic phenotype should decrease the local antibiotic concentration requirement. Several studies have shown that the minimum biofilm eradication concentration (MBEC) for bacteria in biofilm is 10-1000 times higher than the minimum inhibitory concentrations (MIC) for planktonic bacteria [54-56]. There is considerable interest in leveraging antifouling compounds and materials to enhance the efficacy of antimicrobials.

Cis-2-decenoic acid is a fatty acid messenger produced by Pseudomonas aeruginosa that has been shown to promote the degradation of biofilms and prevent biofilm formation in vitro. It has been shown to be effective at preventing the formation of biofilm by several bacterial species, such as Pseudomonas aeruginosa, Klebsiella pneumoniae, Proteus mirabilis, Streptococcus pyogenes, Staphylococcus aureus, and interestingly, the eukaryote Candida albicans [57]. Cis-2-decenoic acid appears to act as a diffusible signaling molecule that allows biofilm organisms to coordinate gene expression via cell-cell communication. Jennings et al. [58] demonstrated in vitro that antibiotics are more effective at preventing biofilm growth when combined with cis-2decenoic acid. Practically, cis-2-decenoic is challenging to incorporate into a sustained release local delivery vehicle since it is a fatty acid. Chitosan sponges have been investigated as a carrier due to their lipid-binding properties, but similar to antibiotic-loaded chitosan, this strategy is limited by short-lived release dominated by burst release kinetics.

Zwitterionic polymers show promise as a novel type of coating that can combine antimicrobial action with non-fouling of implant materials. Cationic polymers are known to be toxic to microbes via interactions with the negatively charged plasma membranes of bacteria, although there is concern that these cationic polymers could be toxic host cell membranes as well $[59,60]$. Another significant disadvantage of these polymers is that dead bacteria are retained through electrostatic interactions. Zwitterionic polymers are known to have non-fouling properties and have been used extensively to deter the attachment of bacteria and the growth of biofilms. Cheng et al. [61] designed a switchable polymer that is toxic to bacteria on contact, then switches to a nonfouling zwitterionic coating to release the debris and maintain a non-fouled surface. Tan et al. [62] have also developed a chitosan derivative with a series of substituted quaternary ammonium groups that can be loaded into PMMA to decrease fouling by antibiotic-resistant Staphylococcus aureus.
$D$-amino acids have become increasingly popular as a signaling molecule for biofilm dispersal. Several studies have noted that $D$-amino acids can both prevent biofilm formation and enhance detachment [63-65]. $D$-amino acids are thought to be synthesized by biofilm bacteria when they reach a critical concentration in order to signal bacteria to detach and colonize a distant site, and the local concentration of $D$-amino acids necessary to signal bacteria to detach is on the micromolar order. The most studied $D$-amino acids for biofilm dispersal are $D$-tyrosine, $D$-leucine, $D$-methionine, and $D$-tryptophan. Each amino acid has a specific activity, but combinations of $D$-amino acids are found to be the most potent against biofilms [64]. $D$-amino acids are inexpensive and have great potential for incorporation into local delivery vehicles, contributing to their current prevalence in the literature.

\section{Conclusions}

The success of tissue engineering strategies will depend on their ability to adequately address infectious complications. Regeneration and repair of tissue cannot occur in the presence of infection, so an effective method of eradicating and preventing infections must be developed. The most robust strategies will address immediate infection and prevent later failure of the implant due to delayed infection by preventing biofilm formation. One method to achieve this is to develop local delivery vehicles for antibiotics and anti-biofilm agents that provide controlled, sustained release, do not themselves host biofilm formation, and can accommodate the emergence of new antibiotics.

This work was supported by the Armed Forces Institute of Regenerative Medicine (W81XWH-08-2-0032).

1 Moyad T F, Thornhill T, Estok D. Evaluation and management of the infected total hip and knee. Orthopedics, 2008, 31: 581-590

2 Hanssen A D, Rand J A. Evaluation and treatment of infection at the site of a total hip or knee arthroplasty. Instr Course Lect, 1999, 48: $111-122$

3 Scott R D. National Center for Preparedness, Detection, and Control of Infectious Diseases (Centers for Disease Control and Prevention). Division of Healthcare Quality Promotion. The direct medical costs of healthcare-associated infections in US hospitals and the benefits of prevention, 2009

4 Gristina A G. Biomaterial-centered infection: Microbial adhesion versus tissue integration. Science, 1987, 237: 1588-1595

5 Gristina A G, Shibata Y, Giridhar G, et al. The glycocalyx, biofilm, microbes, and resistant infection. Semin Arthroplasty, 1994, 5: 160170

6 Bayston R, Ashraf W, Barker-Davies R, et al. Biofilm formation by propionibacterium acnes on biomaterials in vitro and in vivo: Impact on diagnosis and treatment. J Biomed Mater Res, 2007, 81A: 705-709

7 Spellberg B, Guidos R, Gilbert D, et al. The epidemic of antibiotic-resistant infections: A call to action for the medical community from the Infectious Diseases Society of America. Clin Infect Dis, 2008, 46: 155-164 
8 Somayaji S N, Ritchie S, Sahraei M, et al. Staphylococcus aureus induces expression of receptor activator of NF-B ligand and prostaglandin E2 in infected murine osteoblasts. Infect Immun, 2008, 76: 5120-5126

9 Tabel G, Hoa N T, Tarnawski A, et al. Helicobacter pylori infection inhibits healing of the wounded duodenal epithelium in vitro. J Lab Clin Med, 2003, 142: 421-430

10 Schierle C F, De la Garza M, Mustoe T A, et al. Staphylococcal biofilms impair wound healing by delaying reepithelialization in a murine cutaneous wound model. Wound Repair Regen, 2009, 17: 354-359

11 Guo S, DiPietro L A. Factors affecting wound healing. J Dent Res, 2010, 89: 219-229

12 Bost K L, Ramp W K, Nicholson N C, et al. Staphylococcus aureus infection of mouse or human osteoblasts induces high levels of interleukin-6 and interleukin-12 production. J Infect Dis, 1999, 180: 1912-1920

13 Penn-Barwell J G, Murray C K, Wenke J C. Early antibiotics and debridement independently reduce infection in an open fracture model. J Bone Joint Surg Br Vol, 2012, 94B: 107-112

14 Koyonos L, Zmistowski B, Della Valle C J, et al. Infection control rate of irrigation and débridement for periprosthetic joint infection. Clin Orthop Rel Res, 2011, 469: 3043-3048

15 Odum S M, Fehring T K, Lombardi A V, et al. Irrigation and debridement for periprosthetic infections. J Arthroplasty, 2011, 26: 114-118

16 Fehring T K, Odum S M, Berendm K R, et al. Failure of irrigation and débridement for early postoperative periprosthetic infection. Clin Orthop Rel Res, 2013, 471: 250-257

17 Rathbone C R, Cross J D, Brown K V, et al. Effect of various concentrations of antibiotics on osteogenic cell viability and activity. $\mathrm{J}$ Orthop Res, 2011, 29: 1070-1074

18 Wachol-Drewek Z Z, Pfeiffer M M, Schol E E. Comparative investigation of drug delivery of collagen implants saturated in antibiotic solutions and a sponge containing gentamicin. Biomaterials, 1996, 17: 1733-1738

19 Noel S P, Courtney H, Bumgardner J D, et al. Chitosan films: A potential local drug delivery system for antibiotics. Clin Orthop Rel Res, 2008, 466: 1377-1382

20 Mi F L, Wu Y B, Shyu S S, et al. Control of wound infections using a bilayer chitosan wound dressing with sustainable antibiotic delivery. J Biomed Mater Res, 2001, 59: 438-449

21 Kent M E, Rapp R P, Smith K M. Antibiotic beads and osteomyelitis: Here today, what's coming tomorrow? Orthopedics, 2006, 29: 599-603

22 Wenke J C, Owens B D, Svoboda S J, et al. Effectiveness of commercially-available antibiotic-impregnated implants. J Bone Joint Surg Br Vol, 2006, 88: 1102-1104

23 Anagnostakos K, Hitzler P, Pape D, et al. Persistence of bacterial growth on antibiotic-loaded beads: Is it actually a problem? Acta Orthop, 2008, 79: 302-307

24 Turner T M, Urban R M, Hall D J, et al. Local and systemic levels of tobramycin delivered from calcium sulfate bone graft substitute pellets. Clin Orthop Rel Res, 2005, 437: 97-104

25 Cai X, Han K, Cong X, et al. The use of calcium sulfate impregnated with vancomycin in the treatment of open fractures of long bones: A preliminary study. Orthopedics, 2010, 33: 152-157

26 Helgeson M D, Potter B K, Tucker C J, et al. Antibiotic-impregnated calcium sulfate use in combat-related open fractures. Orthopedics, 2009, 32: 323

27 Shi M, Kretlow J D, Nguyen A, et al. Antibiotic-releasing porous polymethylmethacrylate constructs for osseous space maintenance and infection control. Biomaterials, 2010, 31: 4146-4156

28 Buschle-Diller G, Cooper J, Xie Z, et al. Release of antibiotics from electrospun bicomponent fibers. Cellulose, 2007, 14: 553-562

29 Özalp Y, Özdemir N, Kocagöz S, et al. Controlled release of vancomycin from biodegradable microcapsules. J Microencapsul, 2001, 18: 89-110

30 Pillai R R, Somayaji S N, Rabinovich M, et al. Nafcillin-loaded PLGA nanoparticles for treatment of osteomyelitis. Biomed Mater,
2008, 3: 034114

31 Ambrose C G, Gogola G R, Clyburn T A, et al. Antibiotic microspheres: Preliminary testing for potential treatment of osteomyelitis. Clin Orthop Rel Res, 2003, 415: 279-285

32 Ramchandani M, Robinson D. In vitro and in vivo release of ciprofloxacin from PLGA 50:50 implants. J Control Release, 2998, 54: $167-175$

33 Rui J, Dadsetan M, Runge M B, et al. Controlled release of vascular endothelial growth factor using poly-lactic-co-glycolic acid microspheres: In vitro characterization and application in polycaprolactone fumarate nerve conduits. Acta Biomater, 2012, 8: 511-518

34 Ruhé P Q, Boerman O C, Russel F G, et al. Controlled release of rhBMP-2 loaded poly(dl-lactic-co-glycolic acid)/calcium phosphate cement composites in vivo. J Control Release, 2005, 106: 162-171

35 Klasen H J. Historical review of the use of silver in the treatement of burns. I. Early uses. Burns, 2000, 26: 117-130

36 Huh A J, Kwon Y J. "Nanoantibiotics": A new paradigm for treating infectious diseases using nanomaterials in the antibiotic resistant era. J Control Release, 2011, 156: 128-145

37 Davis I J, Richards H, Mullany P. Isolation of silver- and antibiotic-resistant Enterobacter cloacae from teeth. Oral Microbiol Immunol, 2005, 20: 191-194

38 Feng Q L, Wu J, Chen G Q, et al. A mechanistic study of the antibacterial effect of silver ions on Escherichia coli and Staphylococcus aureus. J Biomed Mater, 2000, 52: 662-668

39 Gong P, Li H, He X, et al. Preparation and antibacterial activity of $\mathrm{Fe}_{3} \mathrm{O}_{4} @$ Ag nanoparticles. Nanotechnology, 2007, 18: 285604

40 Rai M, Yadav A, Gade A. Silver nanoparticles as a new generation of antimicrobials. Biotechnol Adv, 2009, 27: 76-83

41 Grewal J S, Tiwari R P. Resistance to metal ions and antibiotics in Escherichia coli isolated from foodstuffs. J Med Microbiol, 1990, 32 : 223-226

42 Choi $\mathrm{S}$, Isaacs $\mathrm{A}$, Clements $\mathrm{D}$, et al. De novo design and in vivo activity of conformationally restrained antimicrobial peptides. Proc Natl Acad Sci USA, 2009, 106: 6968-6973

43 Liu D, Choi S, Chen B, et al. Nontoxic membrane-active antimicrobial arylamide oligomers. Angew Chem Int Ed, 2004, 43: 1158-1162

44 Kazemzadeh-Narbat M, Kindrachuk J, Duan K, et al. Antimicrobial peptides on calcium phosphate-coated titanium for the prevention of implant associated infections. Biomaterials, 2010, 31: 9519-9526

45 Stallman H P, Faber C, Bronckers A L J J, et al. Osteomyelitis prevention in rabbits using antimicrobial peptide hLF1-11- or gentamicin-containing calcium phosphate cement. J Antimicrob Chemother, 2004, 54: 472-476

46 Stallman H P, de Roo R, Faber C, et al. In vivo release of the antimicrobial peptide hlf1-11 from calcium phosphate cement. J Orthop Res, 2008, 26: 531-538

47 Shukla A, Fleming K E, Chuang H F, et al. Controlling the release of peptide antimicrobial agents from surfaces. Biomaterials, 2010, 31: 2348-2357

48 Zasloff M. Antimicrobial peptides of multicellular origin. Nature, 2002, 415: 389-395

49 Bell G, Gouyon P H. Arming the enemy: The evolution of resistance development and persistence. Microbiology, 2003, 149: 1367-1375

50 Perron G G, Zasloff M, Bell G. Experimental evolution of resistance to an antimicrobial peptide. Proc R Soc B-Biol Sci, 2006, 273: 251-256

51 Anaya-López J L, López-Meza J E, Ochoa-Zarzosa A. Bacterial resistance to cationic antimicrobial peptides. Crit Rev Microbiol, 2012, 38: $1-16$

52 Neut D, van de Belt H, Stokroos I, et al. Biomaterial-associate infection of gentamicin-loaded PMMA beads in orthopaedic revision surgery. J Antimicrob Chemother, 2001, 47: 885-891

53 Anagnostakos K, Hitzler P, Pape D, et al. Persistence of bacterial growth on antibiotic-loaded beads: Is it actually a problem? Acta Orthop, 2008, 79: 302-307

54 Olson M E, Ceri H, Morck D W, et al. Biofilm bacteria: Formation and comparative susceptibility to antibiotics. Can J Vet Res, 2002, 66: 86-92 
55 Davies D. Understanding biofilm resistance to antibacterial agents. Nat Rev Drug Discov, 2003, 2: 114-122

56 Kirby A E, Garner K, Levin B R. The relative contributions of physical structure and cell density to the antibiotic susceptibility of bacteria in biofilms. Antimicrob Agents Chemother, 2012, 56: 2967-2975

57 Davies D G, Marques C N. A fatty acid messenger is responsible for inducing dispersion in microbial biofilms. J Bacteriol, 2009, 191: 1393-1403

58 Jennings J A, Courtney H S, Haggard W O. Cis-2-decenoic acid inhibits s. aureus growth and biofilm in vitro: A pilot study. Clin Orthop Rel Res, 2012, 470: 2663-2670

59 Lü H, Zhang S, Wang B, et al. Toxicity of cationic lipids and cationic polymers in gene delivery. J Control Release, 2006, 114: 100-109

60 Nagamune H, Maeda T, Ohkura K, et al. Evaluation of the cytotoxic effects of bis-quaternary ammonium antimicrobial reagents on human cells. Toxicol In Vitro, 2000, 14: 139-147

61 Cheng G, Xue H, Zhang Z, et al. A switchable biocompatible polymer surface with self-sterilizing and nonfouling capabilities. Angew Chem Int Ed, 2008, 47: 8831-8834

62 Tan H, Peng Z, Li Q, et al. The use of quaternised chitosan-loaded PMMA to inhibit biofilm formation and downregulate the virulence-associated gene expression of antibiotic-resistant staphylococcus. Biomaterials, 2010, 33: 365-377

63 Hochbaum A I, Kolodkin-Gal I, Foulston L, et al. Inhibitory effects of $D$-amino acids on staphylococcus aureus biofilm development. J Bacteriol, 2011, 193: 5616-5622

64 Kolodkin-Gal I, Romero D, Cao S, et al. D-amino acids trigger biofilm disassembly. Science, 2010, 328: 627-629

$65 \mathrm{Xu} \mathrm{H}$, Liu Y. D-amino acid mitigated membrane biofouling and promoted biofilm detachment. J Membr Sci, 2011, 376: 266-274

Open Access This article is distributed under the terms of the Creative Commons Attribution License which permits any use, distribution, and reproduction in any medium, provided the original author(s) and source are credited. 